\title{
Der Zauberladen
}

\section{Psychodrama trifft auf Magic}

\section{Gabriele Stiegler}

Zusammenfassung: Die Methode des Zauberladens ist ein spielerischer, kreativer Zugewinn auf der Metaphernebene für jede Psychodramaleiterin und jeden Psychodramaleiter. Dieser Beitrag befasst sich mit der Geschichte und der Durchführung des Zauberladens, mit der Rolle der Besitzerin und der Strategie des Tauschhandels. Die Vorzüge des Gruppen-Zauberladens für die Supervision und die Teamentwicklung werden dargestellt.

Schlüsselwörter: Zauberladen · Tauschhandel · Strategie und Ziel ·

Gruppen-Zauberladen als Teamentwicklungsinstrument $\cdot$ Surplus Reality

\section{The magic shop - Psychodrama meets magic}

Abstract: The method of the Magic Shop is a playful and creative gain on the metaphorical level for every psychodrama leader. This article deals with the history and the realization of the Magic Shop, with the role of the shopkeeper and the strategy of the bartering, and the advantages of the Group-Magic-Shop in supervision and team-building.

Keywords: Magic Shop $\cdot$ Bartering $\cdot$ Strategy and goal ·

Group-Magic-Shop as a team-building instrument $\cdot$ Surplus Reality

Online publiziert: 06.02 .2013

(C) Die Autor(en) 2012. Dieser Artikel ist auf Springerlink.com mit Open Access verfügbar.

G. Stiegler $(\bowtie)$

Psychodramaforum Berlin,

Giesebrechtstraße 11, 10629 Berlin, Deutschland

E-Mail: stiegler@psychodramaforum.de 


\section{Erwärmung}

The Magic Shop. Eine Psychodrama-Methode, die verzaubert.

Ja, das ist tatsächlich gemeint, der Magic Shop verzaubert. Diese Methode ist ein spielerischer Zugewinn, ein psychodramatischer Regenbogen der Metaphernarbeit. Im Zauberladen wird die erweiterte Realität, die Marx ,surplus value“ nannte und Moreno „Surplus Reality“ magisch erlebbar. Und so lade ich Sie ein in den Zauberladen, ich begebe mich als Leiterin in diese magische Rolle der Zauberladenbesitzerin.

Heute besuchen wir den Zauberladen, einen imaginären Ort, an dem Sie der imaginären Ladenbesitzerin eine Antwort geben können auf ihre Frage: „Guten Tag meine Dame/mein Herr. Was kann ich für Sie tun?" Doch zunächst stellt sich eine andere Frage: Wussten Sie schon, dass Sie beim Betreten eines Psychodrama-Workshops gleichzeitig die Welt der Bühne betreten? In dieser Welt sind Zeit und Raum nicht wie Sie es sonst kennen. Alles, was Sie erleben, beginnt mit „Es war einmal“", und es gibt genügend Freiraum, um in den Spiegel zu sehen und magische Verwandlungen zu durchleben. In der Welt des Psychodramas erschaffen die Menschen ihre eigene Welt und ihr eigenes Ich. Die Menschen brauchen Träume, die sie bei der Verwandlung der Welt und ihrer selbst inspirieren und unterstützen.

Wenn wir den Zauberladen betreten und hören die Frage: „Was kann ich für Sie tun?“, wagen wir es dann, in unseren inneren Spiegel zu sehen, bis dorthin, wo unsere tiefsten Träume und ureigensten Wünsche ihr verborgenes Dasein fristen? Und wenn wir sie finden, trauen wir uns dann, sie mit anderen zu teilen? Wenn wir uns dazu eingeladen fühlen, auf diese Weise die Frage der Ladenbesitzerin zu verstehen und zu beantworten, tauchen wir ein in eine angenehme Atmosphäre, in der wir uns ebenso spielerisch und verspielt bewegen als wären wir wieder Kinder.

Denken Sie also einen Moment darüber nach, und fragen Sie sich selbst: Will ich wirklich da hineingehen...? Denn wenn Sie den Zauberladen betreten, kann es sein, dass Sie sich für immer verändern. Man weiß nie, was passieren kann, und an dieser Stelle haben Sie noch die Möglichkeit, sich umzudrehen und zu Ihrem normalen Ich zurückzukehren ...! Sind sie noch hier? Also wollen Sie sich auf diese Erfahrung einlassen? Gut. Ich bitte Sie, Ihre normale, erwachsene Reserviertheit draußen zu lassen und mir zu folgen - in die Welt des Zauberladens, des Magic Shops.

Es ist von Bedeutung, sich selbst in die Rolle der Besitzerin des Magic Shops hineinzuversetzen, sich selbst anzuwärmen, die sonstigen Rollen außen vor zu lassen, um ganz auf der Ebene der Metapher zu bleiben. Ich stehe auf der Bühne vor meinem Laden, öffne den Rollladen, schließe die Tür auf und halte einen inneren Monolog: „Heute möchte ich Sie einladen hier einzutreten, dies ist ein ganz besonderer Laden, hier können Sie alles kaufen, was Sie wollen, vorausgesetzt, es ist nichts Materielles. Ich verkaufe immaterielle Dinge, wie Eigenschaften, Fähigkeiten, Charakterzüge, persönliche Qualitäten wie etwa Wärme, Entschiedenheit, Offenheit, innere Gelassenheit. In meinem Laden kommt es zu einem Tauschhandel. Sie können nur in der Währung von Eigenschaften oder Fähigkeiten zahlen. Falls ich das habe, was Sie benötigen, fordere ich von Ihnen eine Gegenleistung. Das Prinzip ist hierbei, dass die Bezahlung der „Ware“ im gleichen Wert sein soll, sie muss nicht von gleicher Art sein. Die Ladenbesetzerin hat die erforderliche Erfahrung, um dies zu beurteilen. Sofern Sie nichts Passendes für sich finden, können Sie den Laden 
auch wieder verlassen. Das ist vollkommen in Ordnung. Hier befinden wir uns also in diesem Zauberladen (Aktionsfläche auf der Bühne), die Regale sind gefüllt mit allem, was das Herz begehrt. Hier haben wir zum Beispiel die Abteilung, in der sich der Mut stapelt. Es gibt die verschiedensten Facetten des Mutes. Es gibt den Mut um mit etwas anzufangen, den Mut um etwas zu beenden und hier den Mut um etwas durchzuhalten. Oder den Mut, um jemandem endlich einmal die Meinung zu sagen.

Die Menschen außerhalb des Ladens können sehen und hören, was im Laden geschieht. Aber die Person, die den Laden betritt, hört und sieht nur das, was im Laden geschieht. Sie sind also jetzt eingeladen, den Laden zu betreten und $\mathrm{zu}$ handeln, einer nach dem anderen, denn hier bedienen ich noch jeden persönlich, eine nach der anderen, schließlich ist das kein Supermarkt, sondern ein Zauberladen.“

\section{Geschichte des Zauberladens}

Moreno (1972) beschreibt den Zauberladen als eine bewährte Methode. Im Vorwort zur dritten Auflage (ebda., S.X-XI) berichtet er als Beispiel von einer Sitzung, die 1948 abgehalten wurde:

Eine junge, depressive Patientin, die nach einem Selbstmordversuch zum Kurs von 1948 zugelassen worden war, betrat den Zauberladen, mit der Bitte um ,innere Ruhe“. Der Ladenbesitzer, Justus Randolph, ein junger, sensibler Therapeut fragte sie, welche Gabe/Eigenschaft sie im Gegenzug würde eintauschen wollen. „Sie wissen, dass wir Ihnen ohne Ihrer Bereitschaft etwas zu opfern, nichts geben können.“ Auf die Gegenfrage der Patientin, was der Ladenbesitzer haben wolle, forderte dieser etwas, was sie nicht bereit war zu geben: „Nein, der Preis ist zu hoch, um ihn zu bezahlen. Ich möchte dann keine ,innere Ruhe“ haben. “ Sie verließ den Laden und setzte sich auf ihren Platz. Der Ladenbesitzer hatte einen sensiblen Punkt getroffen.

Moreno schreibt, dass der Gruppenleiter entscheiden kann, ob er selbst die Rolle des Ladenbesitzers übernimmt, oder ob er ein Mitglied der Gruppe bittet dieses zu tun.

In den späten 40er und den 50er Jahren ist die Zauberladen-Methode von Hanna B. Weiner (New York), Leon Fine (Saint Louis), Anne Ancelin Schützenberger (Paris) und vielen anderen weiter entwickelt worden. Die Arbeit mit dieser Art der symbolischen Erfüllung von Wünschen ermutigt die Gruppe, in einer spontaneren und kreativeren Art und Weise zu handeln. Deshalb kann die Technik auch als Aufwärmübung, als FeedbackInstrument, in der Teamentwicklung und der Teamsupervision eingesetzt werden.

\section{Strategie und Ziel des Zauberladens}

Zwei Aspekte sind es im Besonderen, dass der Zauberladen sich solcher Beliebtheit erfreut. Er macht es möglich, alle Teilnehmerinnen mit ihren Bedürfnissen anzusprechen, selbst wenn diese völlig verschiedene Wünsche haben. Fast jedes Gruppenmitglied kann etwas für sich Bedeutsames aus dieser Erfahrung mitnehmen. Des Weiteren ist der integrative Charakter der Methode attraktiv. Es wird jedem die Möglichkeit geboten, etwas 
in die Gruppe einzubringen, was sie oder er über sich erfahren hat und nach Wegen zu suchen, wie dies in nützlicheres und produktiveres Verhalten umgesetzt werden kann. Der Handel regt Selbstreflexion an und ist ein Schritt zu persönlichem Wachstum. Der Zauberladen verbindet intensive Gruppenerfahrung mit der Möglichkeit, etwas davon mit nach Hause zu nehmen, um es im Sinne des persönlichen Wachstums im eigenen Leben anzuwenden. Die Methode gibt der Gruppe die Erlaubnis, mit den anderen die befreiende Wirkung des Ganz-Werdens zu teilen, was umso bemerkenswerter ist, als sie ja auf einem anderen Boden als dem der Realität stattfindet. „Ganz im Gegenteil, schließlich ist der gemeinschaftlich anerkannte Schauplatz die Phantasie, die Mehr-als-Wirklichkeit" (Barbour 1992, S.91-99).

Im klassischen Zauberladen-Setting schlüpft die Psychodramaleitung in die Rolle der Ladenbesitzerin. Wie eingangs beschrieben, ist es wichtig sich selbst diese Rollen-Anwärmung zu gönnen, je authentischer ich in der Rolle bin, desto überzeugender für die Kundinnen, dazu gehört auch die Vorstellung des Ladens, der Regale, mögliche Inhalte. Eine gute Anmoderation des Ladens macht neugierig. Im Laden wird dann mit der Methode des ,inneren Monologs“ darauf hingewiesen, dass weder Geld, noch Auto oder Doktortitel erworben werden kann, sondern dass persönliche Qualitäten, Charakterzüge und Fähigkeiten getauscht werden. Oft dauert es einige Minuten, bis die erste Person den Laden betritt. Die Gruppe wartet neugierig ab, ob die Ladenbesitzerin auch tatsächlich im Laden bleibt, egal was außerhalb der imaginären Wände vor sich geht. Es empfiehlt sich, die einzelnen Tauschgeschäfte relativ kurz zu halten, um die Aufmerksamkeit der Gruppe zu behalten. Der Zauberladen ist wie eine Vignettenarbeit, kurz und fokussiert. Ich bleibe übrigens grundsätzlich per Sie im Zauberladen, auch wenn ich die Teilnehmerinnen und Teilnehmer gut kenne.

Der Kunde kommt mit dem inneren Bedürfnis in den Laden und fragt nach einer Eigenschaft. Die Ladenbesitzerin erforscht nun mit dem Kunden seinen Wunsch: für was diese Eigenschaft benötigt wird, wie viel davon, in welcher Form er das benötigt, wo diese Eigenschaft angewendet werden soll, für welche Gelegenheiten bzw. welche Personen wird sie benötigt. Nun ist die Gegengabe an der Reihe. An diesem Punkt wird sehr oft um den Preis gefeilscht. Grundsätzlich sollten folgende Regeln eingehalten werden:

1. Die Kundinnen fragen nach dem, was sie gerne hätten.

2. Es kann nicht mit Geld bezahlt werden, stattdessen mit etwas, von dem die Kundinnen viel besitzen (Fleiß, Ehrgeiz) oder etwas, dem sie hohen Wert beimessen (Unabhängigkeit, Erfolg).

Die Ladenbesitzerin kann folgende Bereiche erkunden:

a. Der Bereich der Schwierigkeit, das Anliegen.

b. Der Preis für die Gabe. Idealerweise sollten das, was erworben wird, und das, was eingetauscht wird, zueinander passen und im Verhältnis zueinander stehen.

c. Zukunftsprobe mit der neuen Eigenschaft. Wie wird sie integriert und wie sieht dann das Leben im Alltag aus? (Abb. 1). 
Abb. 1: Schema des möglichen Ablaufs des Zauberladens nach Barbour (1992)
Zauberladen hat persönliche Qualitäten, Wesenszüge und andere Eigenschaften in den Regalen

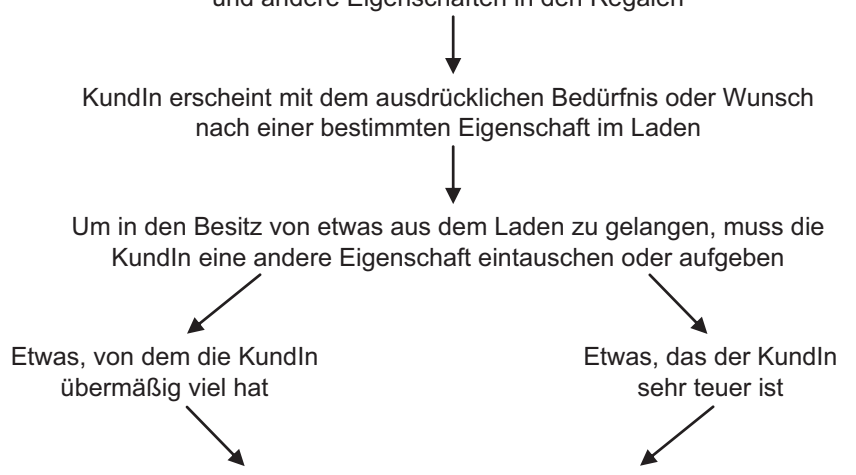

Die Kundln erhält die neue Eigenschaft, nachdem er oder sie den Handel perfekt gemacht hat

\section{Die Zauberin, der Zauberer und die Strategie des Tausches}

Der Zauberladen kommt so leicht daher. In der Umsetzung des Tauschgeschäftes erfordert es allerdings ein gutes Gespür für die Kundinnen, im Hinblick darauf was sie erhalten wollen und das, was sie dafür aufgeben müssen. Die Methode fällt aus dem Rahmen des Gewöhnlichen, weil es der Kunst der Zauberin oder des Zauberers bedarf, wie um den Tausch gehandelt wird. Oft kommt es vor, dass die Kundinnen am Anfang eine Eigenschaft verlangen und mit einer anderen den Laden verlassen. Es ist das erfragen nach dem „Subtext“, was steckt hinter dem Wunsch, was will damit erreicht werden und was muss dafür aufgegeben werden. Hier kann intensiv gefragt werden, wie sich mit dieser Eigenschaft die Rollen verändern, das Umfeld reagiert und wie ernsthaft sie diese benötigen. Was wäre der Benefit, der Fortschritt für die Person? Die Methode erlaubt den Teilnehmerinnen im Zauberladen zu erscheinen, einen Mangel oder einen Bedarf zu äußern, einen Wunsch oder ein Wollen. Dies ist die Herausforderung bei Verfahren wie dem Zauberladen und Vignetten, es sollte versucht werden, diesen Punkt wirklich zu verstehen, um später verhandeln zu können, was aufgegeben werden muss (Abb.2).

Die große Frage für die Zauberin und den Zauberer ist: „Was gibt der Kunde, die Kundin, um sich weiter zu entwickeln?“"

Herr Sauer möchte seine Skepsis anderen gegenüber aufgeben, dafür will er Offenheit und Zuversicht einkaufen. Doch etwas Skepsis ist gut, Offenheit sollte nicht überall und stets gezeigt werden, das könnte ihn in Schwierigkeiten bringen. Herr Sauer könnte auch empathisch, freundlich und geduldig sein. Das hört sich alles gut an, hält ihn allerdings davon ab, etwas anderes auszubilden, das er gerne hätte, zum Beispiel sich abzugrenzen.

Abb. 2: Wahlmöglichkeiten der Kundin nach Barbour (1992)
Als Kundln im Zauberladen kannst Du Dich entscheiden, etwas aufzugeben,

von dem Du übermäßig viel hast (was Dir Schwierigkeiten macht). das Dir sehr teuer ist (das aber Dein persönliches Wachstum hemmt). 
Es ist für die Kundinnen oft ein Problem etwas aufzugeben, das so große Bedeutung hat, aber es kann unumgänglich sein, wenn man dafür etwas anderes erhalten will. Darin liegt auch oft das Paradox, das im Zauberladen zutage tritt, weil der Zauberladen als integrative Aktivität sich mit persönlichem Wachstum beschäftigt, mit Erfüllung und Selbstverwirklichung. Manche von uns hoch geschätzten Eigenschaften sind gleichzeitig die Stolpersteine, die uns im Wege liegen. „Dieses Paradox ist keine neue Vorstellung. Die Griechen sprachen von arete (Vorzug, Tugend) und dem Gegenstück dazu, hamartia (verderbliche Eigenschaft). Nach den alten Griechen sind unsere Problemfelder nicht der Gegensatz zu unseren Tugenden, sondern die uns verderblichen Eigenschaften müssen wir eben in unseren Stärken suchen, unseren ausgezeichneten Charakterzügen“" (Barbour 1992, k.A.). Das Handeln um den Preis im Zauberladen ist eine gute Gelegenheit, solche Stärken und Schwächen näher anzuschauen. Was besitzt die Kundin, hat sie zuviel davon oder misst sie der Eigenschaft großen Wert bei. Eventuell sind die Mängel, die wir an uns finden womöglich bisher nicht geschätzte Stärken. Schön ist es, sich im Zauberladen bewusst werden zu können über unsere Wahrnehmungsweisen und Wertmaßstäbe und sie eventuell infrage zu stellen. Was als Schwäche empfunden wird, kann eine andere Person als Stärke sehen.

\section{5 ,Die revolutionäre Tat ist, zu sagen, was ist ${ }^{66}$}

Es gilt, im Zauberladen in einer eleganten Art, charmant die Wahrheit zu benennen. Die Kundin sitzt vor uns mit unverwechselbarer Persönlichkeit, mit Vorzügen, die ihn oder sie auszeichnen und anderen Eigenschaften, die ihm oder ihr fehlen. So, wie Herr Sauer geschätzt wird wegen seiner Genauigkeit, so wird er weniger geschätzt wegen seiner Fehlerunfreundlichkeit. Sind wir mit einem Antreiber ausgestattet, der es allen recht machen will, fehlt uns eventuell die Konfliktfähigkeit. Oder sind wir auf die Regeln bedacht, fehlt uns vielleicht eine Portion Spontaneität. Es ist nicht so sehr die charakterliche Eigenschaft selbst, um die es geht, als vielmehr die Art, wie sie wahrgenommen und interpretiert wird durch den eigenen Filter und den Filter der Anderen. Im Zauberladen haben wir die Chance zur Begegnung mit dem, wovon wir viel besitzen, was uns lieb und teuer ist, uns aber auch blockiert. Manchmal wollen die Kundinnen auch etwas los werden, das sie blockiert, die Frage ist dann, wie wir das als Zauberer interpretieren.

Eine Kundin wollte einmal ihre Gerührtheit loswerden, bei vielen Gelegenheiten war sie so gerührt, dass sie anfing zu weinen. Diese Gerührtheit habe ich gerne entgegengenommen. Ihr wurde eine Tränenrutsche angesetzt, mit der die kostbaren Tränen aufgefangen wurden. Auf die Frage der Kundin, wem ich die wohl verkaufen könnte, gab ich die Antwort, dass es viele Menschen gibt, die sich nicht mehr anrühren lassen. Ich kann sie an so manchen Kunden verkaufen, der hier eintritt und schon lange nicht mehr geweint hat, wenig empfindet, nicht mehr traurig sein kann. Die Kundin sah in diesem Moment ihre Gerührtheit mit anderen Augen, als kostbares Gut für eine andere Person.

Bei der Auseinandersetzung im Zauberladen geht es weniger um die positive oder negative Eigenschaft, sondern um die Selbst- und Fremdwahrnehmung. Es geht darum, die blinden Flecke zu polieren, wie es Luft und Ingham (1955) so schön in ihrem JohariFenster beschreiben. Beziehungsweise geht es auch um das Quäntchen zu viel oder zu 
wenig. Das ist in der folgenden Prozedur ein guter Maßstab für die Menge der Eigenschaft, die wir anbieten bzw. von der Kundin nehmen.

\section{Die Zukunftsprobe - Surplus Reality}

Die Kundin hat die Möglichkeit, in der Surplus Reality die neue Eigenschaft auszuprobieren Das kann in einer kurzen Szene mit dem Co-Zauberer oder der Co-Zauberin oder einer Teilnehmerin geschehen, die oder der kurz als Hilfs-Ich dient. Gerne benutze ich im Zauberladen auch den Chorus ${ }^{1}$ bei der Zukunftsprobe. Bei manchen Kundinnen sitzt ein alter Glaubenssatz als Blockade fest im Gedächtnis. Nachdem dieser umprogrammiert wurde, in einen hilfreichen und inspirierenden Satz, fordere ich die Gruppe auf, diesen Satz als Chor zu singen, in unterschiedlichen Melodien, bis unsere Kundin diesen neuen Satz im Ohr hat und ihn mitschwingend singt.

In der Rolle als Zauberladenbesitzerin führe ich die Gruppe als ,einen alten bekannten Chor des Zauberladens" ein, den ich vor den Zauberladen bitte, um den Satz einmal gesungen hören zu können. Das wird meist als schöne, aktivierende Teilnahme am Prozess erlebt.

\section{Feedbackphase}

Normalerweise gibt es im Zauberladen keine im Psychodrama übliche Feedbackphase. Doch mache ich am Ende des Tages eine kleine Sharingrunde und frage die Teilnehmerinnen, was sie auch gerne gehabt hätten, oder gerne abgeben würden, was sie mit den Zauberladenkundinnen teilen. Dadurch entsteht eine schöne Atmosphäre (Gruppenkatharsis), die Teilnehmerinnen werden angeregt, am nächsten Tag oder in der nächsten Sequenz den Zauberladen auch einmal zu besuchen. Und vor allem ist es für die Zauberladenkundinnen eine Integration in die Gruppe.

\section{Der Zauberladen als Feedback-Instrument in der Supervision, Team- und Personalentwicklung}

Das Ziel des Zauberladens in der Gruppe ist es, den Teilnehmerinnen eines Seminars oder in der Supervision eine persönliche Rückmeldung aller Beteiligten über ihre Eigenschaften und Fähigkeiten zu geben. Bei einer Gruppe von etwa zehn Teilnehmerinnen wird ungefähr eine Stunde für die Einstimmung, die Durchführung und die anschließende Reflexionsphase benötigt.

\subsection{Einstimmung durch die Leitung}

„Dies ist eine ganz besondere Feedbackrunde. Setzen Sie sich bequem hin. Wenn Sie möchten, schließen Sie die Augen. Stellen Sie sich vor, Sie alle sitzen nicht hier. Sie befinden sich an einem Ort, der weit weg ist. Sie sind in einem Zauberwald.

Es ziehen angenehme Gerüche von Tannen, frischem, saftigem Moos und Waldgrün an Ihnen vorbei. Sie sehen ungewöhnliche Bäume. Sie begegnen außergewöhnlichen Wald- 
bewohnern. Bleiben Sie dort und schauen sich in dem Wald um. Was nehmen Sie wahr? Was sehen Sie? (Lassen Sie den Teilnehmerinnen etwas Zeit, um ihre eigenen Bilder zu bekommen).

Jetzt gehen Sie ein Stück weiter. Sie sehen einen hellen Lichtblick. Gehen Sie in dieser Richtung weiter. Stellen Sie sich vor, Sie finden einen kleinen Zaubermarktplatz - inmitten des Waldes. Um jenen Marktplatz herum stehen kleine, hübsche Läden.

Suchen Sie sich einen Laden aus - einen, der Ihnen richtig gut gefällt. Sie sind jetzt ein Ladenbesitzer, eine Ladenbesitzerin in diesem Zauberwald. Sie haben etwas ganz besonderes zu verkaufen. Sie handeln ausschließlich mit menschlichen Fähigkeiten, mit Eigenschaften. Da das ein ganz außergewöhnlicher Zauberladen ist, nehmen Sie als Besitzerin oder Besitzer kein Geld. Ihr Zauberladen floriert durch Tauschgeschäfte. Das heißt, für jede Fähigkeit, die Sie vergeben, erhalten Sie im Tausch eine andere Fähigkeit. Können Sie sich das schon vorstellen?

- Welche Fähigkeiten könnten Sie anbieten?

- Was zeichnet Sie besonders aus?

- Was sind die Eigenschaften, von denen sie viel haben?

Überlegen Sie auch, von welchen Fähigkeiten Sie unbedingt noch etwas haben möchten. Fähigkeiten die Sie nicht besitzen.

Nun kommen Sie wieder zurück aus Ihrer Phantasie in unser Seminar, der Raum hat sich in den Zauberwald verwandelt. Richten Sie Ihren Laden ein, Sie können ihn auch ausschmücken mit Tüchern oder Gegenständen, die Sie hier finden. Schreiben Sie bitte auf die bereitliegenden Moderationskarten Ihre Fähigkeiten, die Sie auszeichnen und die Sie anbieten wollen.“

\subsection{Durchführung des „Gruppen-Zauberladens“}

Nachdem jeder seinen oder ihren kleinen Laden eingerichtet hat, können die Teilnehmerinnen herumgehen, sich das Angebot anschauen und in den Handel kommen. Wichtig ist, dass es ein „Tauschgeschäft“ wird. Gebe ich die Hälfte meiner Kreativität her (die Moderationskarte wird dann halbiert), ist es wichtig, etwas Gleichwertiges von meiner Tauschpartnerin zu fordern. Dabei ist es auch wichtig zu fragen, in welchem Kontext die Eigenschaft wirkt und wofür ich sie eventuell nicht gebrauchen kann. In der Gruppe beginnt nun ein reges Tauschgeschäft.

\subsection{Reflexionsphase:}

- Welche Rolle ist Dir leichter gefallen: Fähigkeiten anzubieten oder zu erhalten?

- Welchen Eindruck hatte der Handel mit Fähigkeiten auf Dich?

- Welche Fähigkeiten wurden eingetauscht und was bedeuten sie?

Für die Teamentwicklung stelle ich weitere Fragen an das Team:

- Was war hier ein Ladenhüter?

- Was hatten viele/wenige? 
- Was fehlt im Team (z. B. für ein Projekt/zur Konfliktbewältigung)?

- Was könnten Sie als Team noch mit diesen Fähigkeiten bewirken/anfangen (z. B. bei Veränderungsprozessen)?

Der Gruppen-Zauberladen stellt eine erlebnisorientierte Übung für die Teilnehmerinnen dar, die das Selbstbewusstsein stärkt. In vielen Teams liegt das Augenmerk auf dem, was nicht da ist, nicht klappt. Es wird selten der Fokus auf das gerichtet, was alles vorhanden ist, was funktioniert. Dies ist ein schöner Effekt des Gruppen-Zauberladens. Durch dieses „Living-Learning“ verbessert sich außerdem die Qualität des Gruppenprozesses. Die Aktivierung der eigenen Ressourcen kann eine Reorganisation oder Neuorganisation von Handlungsmustern bewirken. Nach dieser Feedback-/Abschlussübung gehen die Teilnehmerinnen sehr euphorisch und motiviert aus dem Seminar oder der Supervision. Es entstehen neue Bilder, neue, veränderte Sichtweisen vom Team und den Teammitgliedern, über Ihr Können und den Gewinn, den die Gruppe nutzen kann.

Der Zauberladen ist ganz besonders geeignet, die psychodramatischen Grundsätze der Kreativität und Spontaneität zu vereinen und sie praktisch, spielerisch werden zu lassen.

Danke für Ihre Aufmerksamkeit und viel Freude beim Zaubern!

\section{Anmerkung}

1 „Die zweite Öffentlichkeit schaffende Instanz, die Moreno neben den Mitspielern benennt, ist die Gruppe, die er mit dem Chor im griechischen Drama vergleicht. Als Publikum des psychodramatischen Spiels und soziometrischen Geschehens tritt sie dem Protagonisten als objektivierende, kritische Instanz gegenüber. Sie wird zum „Resonanzboden der öffentlichen Meinung“, repräsentiert die Welt, zu der der Spieler in Beziehung tritt, und wird dabei umso wichtiger, je einsamer und isolierter der Protagonist ist" (Hutter 2000).

Open Access: Dieser Artikel unterliegt den Bedingungen der Creative Commons Attribution Noncommercial License. Dadurch sind die nichtkommerzielle Nutzung, Verteilung und Reproduktion erlaubt, sofern der/die Originalautor/en und die Quelle angegeben sind.

\section{Literatur}

Barbour, A. (1992). Purpose and strategy behind the magic shop. Journal of Group Psychotherapy, Psychodrama \& Sociometry, 45(3), 91-101.

Hutter, C. (2000). Psychodrama als experimentelle Theologie. Rekonstruktion der therapeutischen Philosophie Morenos aus praktisch-theologischer Perspektive. Münster: LIT.

Luft, J., \& Ingham, H. (1955). The Johari Window. A Graphic Model for Interpersonal Relations. Western Training Laboratory in Group Development. Los Angeles: University of California. Moreno, J. L. (1972). Psychodrama. Vol. I. 4. Aufl. Beacon: Beacon House. 


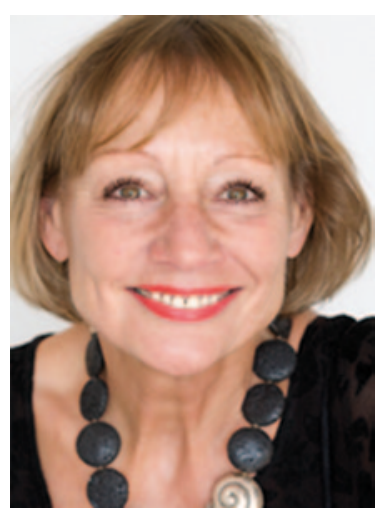

Gabriele Stiegler, 1951, Dipl.-Psychologin, Psychologische Psychotherapeutin, Psychodramatherapeutin (DFP), seit 1994 Psychodrama-Weiterbildungsleiterin, Lehr-Supervisorin (DGSv). 2003 gründete sie das Weiterbildungsinstitut Psychodramaforum Berlin (DFP/FEPTO), sie ist tätig für nationale und internationale Konzerne in der Personal- und Organisationsentwicklung. 